\title{
知行分离视角下郑州市居民低碳行为 影响因素研究
}

\author{
张晶飞 ${ }^{1,2}$, 张丽君 ${ }^{1,2^{*}}$, 秦耀辰 ${ }^{1,2}$, 王 霞 ${ }^{1,2}$, 孙莹莹 ${ }^{1,2}$, 荣培君 ${ }^{3}$ \\ (1. 黄河中下游数字地理技术教育部重点实验室, 河南开封 475004；2. 河南大学环境与规划学院, 河南开封 475004; \\ 3. 河南财经大学旅游与会展学院, 郑州 450052)
}

\begin{abstract}
摘 要: 居民低碳行为-认知研究受到学者们的广泛关注, 而行为-认知分离却是困扰低碳城市建设和居民生活方 式转变的关键问题。论文构建 “空间-行为/认知”分析框架, 采用2018 年郑州市大规模实地调查和遥感影像数据, 运用专家打分法从居家用能、日常出行与生活消费 3 个方面测算 1485 户家庭的低碳行为、认知水平, 使用彩色编码 法从行为一认知偏差视角将低碳行为分为绿色型、被迫型、易感型、红色型, 使用多元Logistic 回归模型分析不同低 碳行为的影响因素。研究发现: (1) 郑州市居民低碳行为存在行为-认知偏差, 4 种低碳行为所占比例差异较大; (2) 绿色型低碳行为主要集聚在土地利用混合度高、基础设施便利的区域, 被迫型、易感型、红色型低碳行为在空间上 呈随机分布; (3) 4 种低碳行为影响因素差异较大。居住区步行指数较高、低碳认知水平高的居民趋于绿色型低碳 行为, 居民低碳行为与低碳认知水平均较高; 居住区住房条件相对较差、居民学历水平低, 居民趋于被迫型低碳行 为, 低碳认知水平低但低碳行为水平较高; 高档住宅居民趋于易感型低碳行为, 居民的低碳认知水平高但低碳行为 水平较低; 经济水平高的年轻居民趋于红色型低碳行为,低碳行为和低碳认知水平均较低。
\end{abstract}

关 键 词: 低碳行为; 建成环境;行为-认知偏差; 多元 Logistic 回归;郑州市

居民碳排放是温室气体排放的主要来源和新 的增长点 ${ }^{[1]}$ 。1992-2013 年, 中国城市居民人均碳 排放量(包括生活用能、日常出行和隐含碳排放)从 $1.72 \mathrm{t}$ 增加到 $10.37 \mathrm{t}$, 平均年增长率为 $8.93 \%$ 。 着城市化进程加快与可支配收人增加, 居民的日常 生活行为逐渐高碳化。低碳行为作为一种资源节 约型、环境友好型行为, 会影响居民的日常生活行 为和消费模式, 进而减少日常活动的温室气体排 放 $^{[3-5]}$ 。因此, 有意识培养居民的低碳行为逐渐成为 地理学、生态学、社会学等多学科共同关注的重点 ${ }^{[6]}$ 。

已有研究基于计划行为理论(theory of planned behavior, TPB) ${ }^{[7-8]}$ 、价值-信念-准则理论(value-belief-norm, $\mathrm{VBN})^{[7-8]}$ 和 $\mathrm{ABC}$ 理论 ${ }^{[7]}$ 研究低碳行为认知 的影响因素, 主要分为以下 4 类: (1) 个人属性。性 别、年龄、学历、职业等会对低碳行为产生影响。家 庭用能存在性别差异, 女性较男性更为积极 ${ }^{[7,9]}$; 年长 者的能源节约意识更强 ${ }^{[9]}$ 。提高教育程度对居民低 碳消费行为认知有积极作用 ${ }^{[10-11]}$ 。职业也对低碳行 为认知产生影响, 即公务员、单位职员的行为认知水 平较高, 学生次之 ${ }^{[12]}$ 。(2) 家庭特征。家庭月收人、 住房类型、私家车数量影响居民低碳行为认知。经 济条件差的居民在日常生活中会倾向节俭化的消费

收稿日期 : 2019-03-08; 修订日期 : 2019-05-05。

基金项目: 国家自然科学基金项目(41671536,41501588);2017年度河南省高等学校重点科研项目(17A170006); 中国博士后科 学基金面上项目(2017M622333)。[Foundation: National Natural Science Foundation of China, No. 41671536 and 41501588; Key Scientific Research Projects of Institutions of Higher Learning in Henan Province in 2017 , No. 17A170006; China Postdoctoral Science Foundation, No. 2017M622333. ]

第一作者简介: 张晶飞(1993-), 女, 河南驻马店人,硕士生, 主要从事居民低碳行为与可持续发展研究。

E-mail: 18211719372@163.com

*通信作者简介:张丽君(1985-),女,河南南阳人,博士,副教授,主要从事区域可持续发展研究。E-mail: zlj7happy@163.com

引用格式: 张晶飞, 张丽君, 秦耀辰, 等. 知行分离视角下郑州市居民低碳行为影响因素研究 [J]. 地理科学进展, 2020, 39(2): 265-275. [Zhang J ingfei, Zhang Lijun, Qin Yaochen, et al. Influencing factors of low-carbon behaviors of residents in Zhengzhou City from the perspective of cognition-behavior gaps. Progress in Geography, 2020, 39(2): 265-275. ] DOI: 10.18306/dlkxjz.2020.02.008 
行为 ${ }^{[7,13-14]}$ 。住房类型也会影响居民的低碳行为认 知, 居住在自有住房的居民低碳行为水平较高 ${ }^{[7]}$ 。 (3) 态度因素。环境意识高的人更易于实施环境友 好的行为 ${ }^{[15-16]}$ 。绿色消费态度会影响居民的消费行 为 ${ }^{[5]}$ 。环境知识正向影响居民消费行为 ${ }^{[10,17-18]}$ 。 (4) 生 活环境。环境政策和社会准则对低碳消费行为产生 正向影响 ${ }^{[7,19-20]}$ 。研究还发现, 居民在低碳行为决策 时, 除考虑环境意识外, 还会考虑舒适、方便、经济 等因素, 居民的态度或认知可能不会产生亲环境行 为, 行为一认知会出现不一致现象, 即存在低碳行 为一认知偏差 ${ }^{[6,21]}$ 。

综上可知, 居民低碳行为影响因素的研究主要 从价值观、规范、态度等主观性视角开展, 也有学者 侧重研究城市空间形态对居民出行行为的约束效 应 $^{[22-25]}$,但尚未顾及空间环境对居民日常生活行为 的作用。此外, 低碳行为研究中较少从行为一认知 偏差视角进行研究, 而在基础设施和城市形态尚未 定型的快速城市化区域, 居民更易存在低碳行为一 认知偏差 ${ }^{[21]}$ 。

基于此, 本文选取郑州市作为研究对象, 从行 为一认知偏差视角构建 “空间-行为/认知” 分析框 架, 采用 2018 年大规模实地调查和遥感影像数据, 运用专家打分法从居家用能、日常出行与生活消费 3 个方面测算 1485 户家庭的低碳行为水平, 然后使 用彩色编码法将其低碳行为分为绿色型、被迫型、 易感型、红色型, 最后基于多元 Logistics 模型分析 不同类型低碳行为的影响因素,进而为郑州市的低 碳城市建设提供一定的参考。

\section{1 分析框架}

\section{1 低碳认知}

意识是个人行为的心理结构和偏好基础 ${ }^{[6,26-27]}$ 。 环境意识是在环境污染的外部刺激下, 由认知系统 驱动而产生的环保行为意愿, 因此, 具有环境意识 的个体更可能实施环保行为并保持行为的持续性 ${ }^{[6]}$ 。

低碳认知作为环境意识的重要组成部分, 是居 民产生低碳行为的重要影响因素 ${ }^{[21]}$ 。低碳认知是 居民对低碳事物和低碳行为的认同和接受程度, 由 个人的知识、价值观和认知驱动 ${ }^{[27]}$, 包括低碳行为 意向、低碳认知水平和低碳认知环境 3 个方面, 其中 低碳行为意向指居民欲实施低碳行为的倾向 ${ }^{[28]}$, 低 碳认知水平是居民对各类低碳行为的了解和认可
程度 ${ }^{[27]}$, 低碳认知环境表示居民在居住环境周围接 触低碳知识的程度和机会。因此人们普遍认为, 低 碳认知与低碳行为呈现正相关, 即居民对低碳生活 方式重要性的认识程度越高, 低碳行为水平也就越 高 ${ }^{[21]}$ 。

\section{2 低碳行为}

低碳行为作为亲环境行为的子集, 是居民为兼 顾保护环境和日常生活,基于低碳认知而采取的保 护生存环境、合理利用资源的行为方式 ${ }^{[29-30]}$ 。其中 “目标-框架理论”认为动机和行为很少是完全统一 的, 大多数情况下呈现多样化、混合化, 甚至相互干 扰的状态, 某一行为可能由不同动机驱动, 而不同 行为也可能由相同动机触发 ${ }^{[6]}$ 。因此, 居民的低碳 行为并非均由低碳认知驱动, 可能受其他多重因素 影响, 比如舒适、方便和经济等,认知与行为并非完 全一致,会存在行为一认知偏差 ${ }^{\left[{ }^{[}\right]}$。

本文基于彩色编码方法,在考虑行为-认知偏 差的基础上, 从低碳行为-认知 2 个维度出发, 将居 民的低碳行为划分为以下 3 种颜色 4 种类型 ${ }^{[6]}$ :

(1) 绿色型低碳行为: 低碳认知和低碳行为水 平均较高。

(2) 红色型低碳行为: 低碳认知和低碳行为水 平均较低。

(3) 灰色型低碳行为: 低碳认知和低碳行为存 在一定偏差。该类型分为易感型低碳行为(居民低 碳认知水平高但低碳行为水平较低)和被迫型低碳 行为(居民低碳认知水平低但低碳行为水平较高)。

\section{3 空间 - 行为/认知}

人类的行为认知建构无法独立于外在情境而 存在, 外在生活环境对居民的行为、认知会产生影 响 ${ }^{[3]}$ 。 $\mathrm{ABC}$ 理论认为居民行为 $\mathrm{B}$ (behavior) 受到居 民态度 $\mathrm{A}$ (attitude) 和外部环境 C(conditions) 的共同 作用, 但更重视外部环境的重要性 ${ }^{[7,32]}$ 。此外, 空间一 行为互动理论认为城市空间形态与居民行为、认知 之间存在互动关系, 空间形态影响居民的日常生 活模式 ${ }^{[23]}$ 。

基础设施与城市建成环境紧密相连,塑造城市 内部土地利用,进而影响居民的交通选择、住房和 行为模式 ${ }^{[1,33]}$ 。城市空间形态是人类活动空间组织 和安排 ${ }^{[34-35]}$, 主要分为都市区尺度和社区尺度。都 市区尺度大多涉及土地利用形态、交通形态和景观 生态形态, 常用城市形态表示; 社区尺度作为城市 建设的基本单元, 更易受到规划者和政策制定者的 
关注,一般指社区形态和城市设计形态, 可用微观 建成环境表示 ${ }^{[36-37]}$ 。本文的基本研究单元为居住 区,因此侧重分析微观建成环境对居民低碳行为的 影响。

基于此, 本研究从低碳行为-认知 2 个维度出 发, 构建 “空间-行为/认知” 分析框架(图 1), 分析微 观建成环境、低碳行为和低碳认知之间的关系。其 中, 微观建成环境涵盖住房环境与居住环境, 社会 经济环境包括家庭属性和个人特征, 低碳行为分为 绿色型、被迫型、易感型和红色型。具体而言, 微观 建成环境对居民的低碳行为具有锁定效应, 即高档 化住房居民的低碳行为可能是红色型或易感型; 普 通单元式住房居民会存在行为一认知偏差; 居住区 步行指数高, 居民可能为绿色型低碳行为。社会经 济属性也会影响居民低碳行为, 即表现为经济水平 高、出行方式高碳化的居民, 可能是易感型或红色 型低碳行为, 可能存在行为-认知偏差; 低学历的工 薪阶层居民为被迫型低碳行为; 住房面积会影响居 民的低碳行为选择; 年龄阶段不同, 居民的低碳行 为也会存在差异。低碳认知水平高的居民可能会 趋于绿色型低碳行为。

\section{2 数据和方法}

\section{1 研究区概况}

郑州作为中原经济区核心, 国家中心城市, 处 于城市化快速发展阶段。尤其是《郑州市城市总体 规划(2010-2020年)》中“一主一城三区四组团”的 城镇布局, 为郑州提供了发展空间。主要表现在:

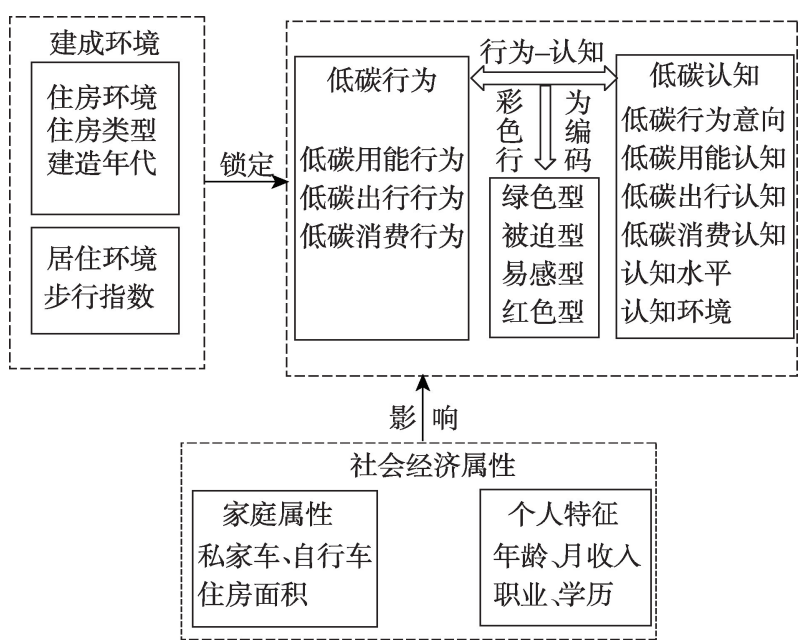

图 1 低碳行为分析框架

Fig.1 A framework for the analysis of low-carbon behaviors
(1) 城市化进程发展迅速。2000-2017年, 城镇人 口由 257.7 万增加到 506 万; 建成区面积增加 2.75 倍, 从 $133.2 \mathrm{~km}^{2}$ 到 $500.8 \mathrm{~km}^{2}$; 城市化率由 $55.1 \%$ 增 加到 $77.2 \%$ 。(2) 环境质量下降。城市化的快速推 进和功能分区规划使得汽车拥有量和职住距离显 著提高, 此外还受产业结构调整和环境破坏等影 响, 空气质量下降、雾䨪加重 ${ }^{[3-39]}$ 。(3) 居民生活行为 方式多样化, 郑州市居民低碳消费认知存在差异 ${ }^{[40]}$ 。

\section{2 数据来源}

本文数据来源主要有 3 类:

(1) 问卷调查数据为基础数据来源。首先进行 问卷预调查。通过网络发放 150 份问卷,初步分析 结果后对问卷稍作修改; 然后, 采取分层随机调查 的方式, 在 2018 年 5 月对研究区域进行大规模实地 调查。依据研究区域的人口分布特征, 分别在一环 到四环发放问卷的 $9.2 \% 、 31.8 \% 、 40.1 \%$ 和 $17.9 \%$, 共 发放 1700 份问卷,剔除部分填写不完整、坐标定位 不明等残缺问卷后,共有 1485 份问卷用于分析, 涉 及 5201 名居民 ${ }^{[41]}$ 。据《2018 年郑州市统计年鉴》, 2017 年郑州市城镇居民为 506 万, 问卷有效抽样比 达到 $0.1 \%$ 左右。问卷调查显示(表 1), 性别构成基 本持平, 男女比例分别为 $49.7 \%$ 和 $50.3 \%$ 。从年龄 构成来看, 中青年(20 50 岁)比例较大, 达到 $72.3 \%$; 婚姻状况以已婚为主; 本科或大专学历所占比重为 $61.8 \%$; 工薪阶层员工居多; 居民月收人 $6000 ~ 12000$ 元所占比例为 $36.8 \%$ 。

(2) 空间数据。空间数据来源于水经注下载谷 歌遥感影像数据(级数 19 级, 空间分辨率为 $0.6 \mathrm{~m}$ ) 和百度 POI(Point of Interest)数据。借助 ArcGIS 和 Envi 提取郑州市主城区遥感影像中的居住区、建 筑、道路等, 以此构建数据库 ${ }^{[4]}$ 。

(3) 社会经济数据。本文社会经济数据来源于 郑州市统计局及历年《郑州市统计年鉴》,包括建成 区面积、城镇人口、城镇化率和汽车拥有量等, 获取 郑州市的总体发展状况。

\section{3 研究方法}

\subsection{1 彩色编码的低碳行为量化}

居民碳排放研究分为直接、出行和隐含 3 类, 据 此, 从用能、出行和消费 3 个方面测算居民低碳认 知、行为水平。依据专家打分法对问卷结果从高到 低赋值为 $9 、 7 、 5 、 3 、 1$, 汇总后求平均值, 保证低碳认 知和低碳行为得分在 $(0,9]$ 之间。

(1) 低碳行为测算: 低碳用能、消费行为分别以 家庭电器使用时长和家庭花销作为基础数据, 计算 
表 1 样本基本特征比例结构

Tab.1 Basic features of the samples

\begin{tabular}{|c|c|}
\hline 变量 & 变量设定与说明 \\
\hline 性别特征 & 男(49.7\%)、女(50.3\%) \\
\hline 婚姻状况 & 未婚(21.8\%)、已婚(75.6\%)、离异或丧偶(2.6\%) \\
\hline 职业状况 & 行政人员(16.5\%)、工薪阶层(62.0\%)、无业或学生(21.5\%) \\
\hline 学历结构 & 初中及初中以下(5.0\%)、高中或中专(21.6\%)、本科或大专(61.8\%)、本科以上(11.6\%) \\
\hline 年龄特征 & $\leqslant 20$ 岁(24.3\%)、20 35 岁(52.8\%)、35 50 岁(19.5\%)、50 岁以上 $(3.4 \%)$ \\
\hline 收人结构 & $\leqslant 6000$ 元/月 $(20.3 \%) 、 6000 \sim 12000$ 元/月(36.8\%)、12000 20000元/月(31.2\%)、>20000元/月 $(11.8 \%)$ \\
\hline
\end{tabular}

各类电器使用时长和家庭花销的平均值 $X_{i}$, 然后按 照 $X_{i}$ 的 $1 、 1.5 、 2 、 3$ 倍作为界限, 分别赋值为 $9 、 7 、 5 、$ $3 、 1$, 最后按照类别数对其求平均值, 测算用能行为 和消费行为水平。低碳出行水平以居民各类出行 活动的出行频率、出行距离、交通方式为基础数据, 依据出行频率赋权重, 按照距离和交通方式赋值 (表2), 测算居民低碳出行水平。低碳出行水平 $S_{c}$ 计算公式为:

$$
S_{c}=\frac{1}{n} \sum_{j=1}^{m}\left(k_{j} \times a_{1 j} \times a_{2 j}\right)
$$

式中: $m$ 为出行活动设计的问卷个数; $n$ 为居民活动 类别数; $k_{j}$ 为第 $j$ 类活动的出行频率权重; $a_{1 j}$ 和 $a_{2 j}$ 分别表示第 $j$ 类活动的出行距离和交通方式赋值。

(2) 低碳认知测算: 低碳用能认知依据居民购 买家电时考虑节能程度和能耗排名, 低碳出行认知 按照首选出行方式中自行车和步行方式的排名, 对 用能认知和出行认知水平按照 $9 、 7 、 5 、 3 、 1$ 赋值; 低 碳消费认知首先根据河南省投人产出表,计算各选 项所对应的相关部门的碳排放强度, 而后结合特尔 菲法赋值, 计算出总得分, 依据得分高低进行赋值 (表3)。

(3) 低碳行为的彩色编码。首先, 根据认知、行 为水平 $(F 、 S)$ 得分, 定义 $F(S) \geqslant 5$ 为认知 (行为) 水平 高; $F(S)<5$ 为认知(行为)水平低。然后, 根据彩色编 码方法, 分别将绿色型 $(F \geqslant 5 、 S \geqslant 5)$ 、被迫型 $(F<5 、 S>$ $=5)$ 、易感型 $(F \geqslant 5 、 S<5)$ 、红色型低碳行为 $(F<5 、 S<$
$5)$, 分别赋值为 $1 、 2 、 3 、 4^{[6]}$ 。

\subsection{2 居住环境指标}

步行指数是衡量居住区步行能力的指标, 表示 所处位置到周围各项生活服务设施(餐饮、购物、教 育、医疗、金融、市政、文体、绿地和个护)之间的便 捷性, 是一种功能主导的计算方法 ${ }^{[42]}$ 。按照美国学 者提出的步行指数(www.walkscore.com)算法,计算 各居民区的步行指数值, 在计算过程中不仅考虑了 日常基础服务设施的种类和空间布局,还考虑其对 居民的相对重要性,并以距离衰减指数、道路交叉 口密度和街区距离进行修正 ${ }^{[22-43]}$ 。由计算过程可 知, 步行指数考虑到交叉口密度、土地利用混合度、 街道设计等,是描述居住区建成环境总体特征的综 合指标 ${ }^{[4]}$, 可作为居住区建成环境的数据来源。计 算公式如下:

$$
W_{s}=\sum_{i=1}^{n} \sum_{j=1}^{m}\left(W_{i} \times S_{i j} \times D_{i j} \times \mathrm{JC}_{s} \times \mathrm{JQ}_{s}\right)
$$

式中: $W_{s}$ 表示 $s$ 小区的步行指数值, $i$ 为不同类型的 服务设施, $j$ 表示不同范围的步行距离, $W_{i}$ 表示某类 设施的权重, $S_{i j}$ 为某类设施不同步行距离的服务范 围, $D_{i j}$ 表示为某类设施在不同距离下的距离衰减指 数, $\mathrm{JC}_{s}$ 表示 $s$ 小区的交叉口密度衰减率, $\mathrm{JQ}_{s}$ 表示 $s$ 小区的街区长度衰减率 ${ }^{[43]}$ 。

\subsection{3 多元Logistic 模型}

Logistic 回归作为一种广义的线性回归分析模 型, 是对线性回归的补充, 分析 $Y$ 取某个值的概率 $P$

表 2 低碳出行水平赋值

\begin{tabular}{|c|c|c|c|c|c|}
\hline 出行频率 & 权重 $k$ & 出行距离 & 交通方式 & 碳排放/(g/km) & 赋值 $a$ \\
\hline 每月 1 次、每年 1 次、每年 2 次、每年 3 次及以上 & 1 & $0 \sim X_{i}$ & 步行、自行车 & 0 & 9 \\
\hline 每月 3 次及以上、每月 2 次 & $7 / 9$ & $X_{i} \sim 1.5 X_{i}$ & 电动车、摩托车 & 8 & 7 \\
\hline 每周 1 次、每周 2 次 & $5 / 9$ & $1.5 X_{i} \sim 2 X_{i}$ & 地铁、其他 & 9.1 & 5 \\
\hline 每天 1 次、每周 3 次及以上 & $3 / 9$ & $2 X_{i} \sim 3 X_{i}$ & 公交车、单位班车、商场巴士 & 35 & 3 \\
\hline 每天 2 次、每天 3 次及以上 & $1 / 9$ & $\geqslant 3 X_{i}$ & 小汽车、出租车 & 135 & 1 \\
\hline
\end{tabular}

Tab.2 Low-carbon travel level value assignment 
表 3 低碳消费认知水平赋值

Tab.3 Low-carbon consumption cognitive level value assignment

\begin{tabular}{llcccc}
\hline \multicolumn{1}{c}{ 问卷结果 } & 消费类别 & 碳排放强度/(t/万元) & 特尔菲法 & 得分 & 赋值 \\
\hline 随手关灯,用完电脑/视后及时关机 & 电 & 10.6052 & 1 & 10.605 & 9 \\
设定合适的空调温度 $\left(\right.$ 冬季 $\leqslant 20^{\circ} \mathrm{C}$, 夏季 $\left.\geqslant 26^{\circ} \mathrm{C}\right)$ & 电 & 10.6052 & 0.7 & 7.4236 & 7 \\
使用太阳能热水器 & 电 & 10.6052 & 1 & 10.605 & 9 \\
节约用水,一水多用 & 水 & 7.0528 & 1 & 7.0528 & 7 \\
少用一次性用具 & 就餐、衣着 & 1.6807 & 0.7 & 1.1765 & 5 \\
购物时自带采购袋 & 食品、衣着 & 1.7037 & 0.7 & 1.1926 & 5 \\
避免无用购物,节俭生活 & 食品、衣着、家电 & 1.8985 & 0.7 & 1.3290 & 5 \\
住房改用双层玻璃 & 家电 & 2.2883 & 0.3 & 0.6865 & 1 \\
换、捐赠、改造多余废旧品 & 食品、家电、衣着 & 1.8985 & 0.5 & 0.9493 & 3 \\
将生活废弃物分类处理 & 食品、家电、衣着 & 1.8985 & 0.5 & 0.9493 & 3 \\
\hline
\end{tabular}

与 $X$ 的关系, 当因变量 $Y$ 的分类超过 2 类时, 可使用 多元 Logistic 模型。本文以居民低碳行为作为因变 量, 使用多元 Logistic 模型分析居民各类低碳行为 的影响因素, 揭示各因素对居民低碳行为的作用强 度, 回归模型如下:

$$
\ln \frac{p_{i}}{1-p_{i}}=\alpha+\sum_{k=1}^{k} \beta_{k} x_{k i}
$$

式中: $p_{i}=P\left(y_{i}=1 \mid x_{1 i}, x_{2 i}, \cdots, x_{k i}\right) ; x_{k i}$ 为自变量, 即影 响居民低碳行为的相关变量; $\beta_{k}$ 为回归系数; $\alpha$ 表 示截距 ${ }^{[39,45]}$ 。

\section{3 特征分析}

\section{1 结构特征}

郑州市居民低碳行为存在行为一认知偏差现象 (比重达到 54\%), 其中低碳认知水平高于低碳行为 水平偏差比例较大 $(46 \%)$; 各类低碳行为比例分布 不均匀, 易感型低碳行为居民所占比重较大, 被迫 型低碳行为居民比重较小(图2)。

从图 2 来看, 郑州市居民各类低碳行为比例分 布不均匀, 其中绿色型、被迫型、易感型、红色型低 碳行为比重分别为 $25 \% 、 8 \% 、 46 \% 、 21 \%$ 。易感型(居 民低碳认知水平高但低碳行为水平较低)所占比例 为被迫型(居民低碳认知水平低但低碳行为水平较 高)的近 6 倍, 差距较大。易感型和被迫型低碳行为 比重占到 $54 \%$, 表明郑州市大多数居民存在低碳行 为-认知偏差现象, 也从侧面说明基于行为一认知偏差 研究低碳行为的必要性。其中易感型和被迫型低 碳行为比重分别为 $46 \% 、 8 \%$, 说明郑州市居民可能 存在认知水平高于行为水平的行为一认知偏差现象。

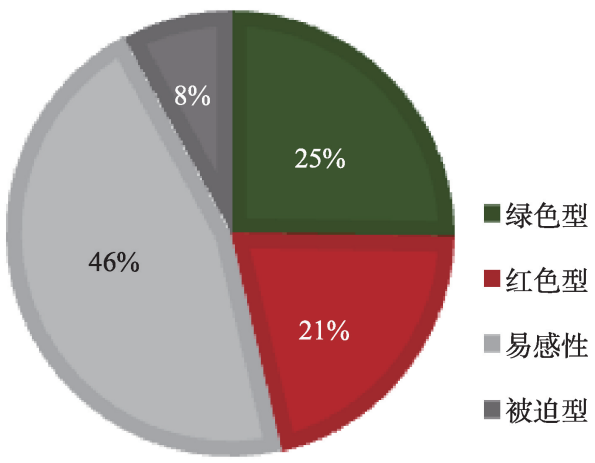

图 2 郑州市居民不同类型低碳行为比例

Fig.2 Proportion of different types of low-carbon behaviors of residents in Zhengzhou City

\section{2 空间特征}

郑州市居民绿色型低碳行为聚集在三环范围 内土地利用混合度较高区域; 被迫型、易感型和红 色型低碳行为在空间内呈现随机分布(图 3)。

绿色型低碳行为分布在三环范围内土地利用 混合度高、基础设施较好的商务、商贸区域,例如: 二七广场、郑东新区、郑州东站等区域。原因主要 有: (1) 三环范围内主要为郑州市老城区, 该区域步 行指数较高, 基础设施较为完善、土地混合度相对 较高, 居住在绿色建成环境居民的低碳行为一认知 差距可能较小, 低碳行为趋于绿色型。(2)二七广 场、郑东新区等区域为郑州市主城区, 以商贸、商务 为主。该区域人流、车流量较大, 道路交通拥堵, 居 民可能倾向于自行车或步行等低碳化但高效率的 出行方式。

易感型低碳行为居民成片分布,但并未有明显 的空间聚集区。在生活服务设施较为完善的主城 区, 即使居民认知水平高, 但可接触的商品服务较 


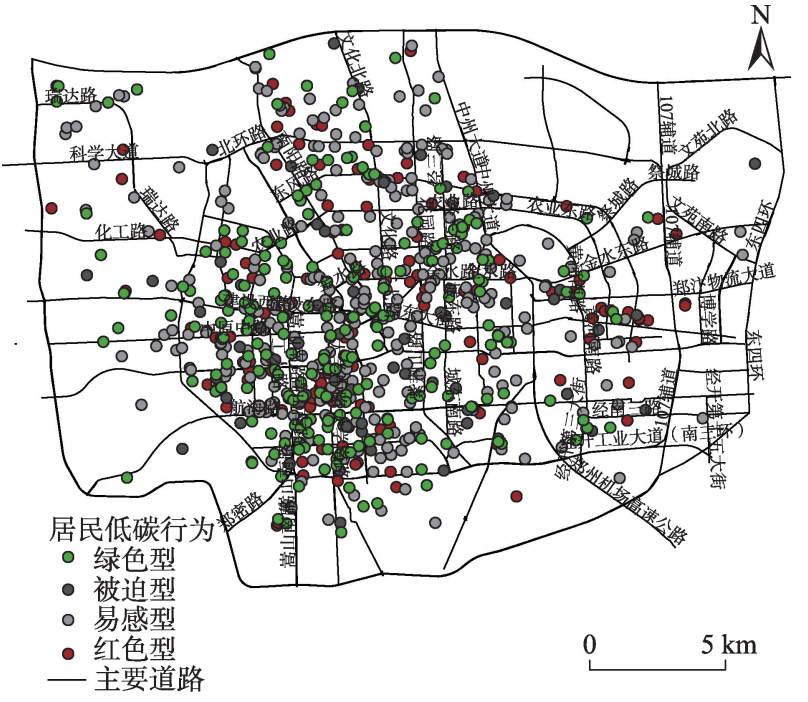

图 3 郑州市居民各类型低碳行为空间分布

Fig.3 Spatial distribution of various types of low-carbon behaviors of residents in Zhengzhou City

多, 消费层次可能较高, 使得居民生活行为方式趋 于高碳化。由此也可看出, 居民日常生活行为受内 外环境综合作用,相同的社区微观建成环境条件可 能存在不同类型的低碳行为, 这就需要综合多方因 素研究低碳行为; 在城市化发展快速区域,各项基 础设施规划布局相对不完善, 居民受基础设施和道 路可达性等外在条件影响, 即使低碳认知水平高, 日常行为可能趋于高碳化。

红色型和被迫型低碳行为居民分散在各区域 内。郑州市老城区内存在文化素质低、无业的居 民, 日常生活行为呈现高碳化, 低碳认知和行为水 平均较低; 但由于老城区基础设施相对完善、道路 通达性较高, 会驱使居民日常行为低碳化。

\section{4 影响因素分析}

先使用相关性检验, 笁选出相关变量; 再将自 变量纳人多元Logistic 回归模型分析各类低碳行为 的影响因素。其中, 表 4 中模型 1 、模型 2 、模型 3 分 别表示绿色型、被迫型、易感型与红色型低碳行为 的对比结果。

\section{1 社会经济属性}

社会经济属性对被迫型低碳行为产生较大影 响(表 4)。与Yang 等研研究结论一致, 年老、高学历、 工薪阶层居民低碳行为水平较高。即表现为低学 历的工薪阶层居民日常行为趋于被迫型; 出行方式 高碳化、住房面积大的年轻居民趋于红色型低碳行
为。

(1) 从个人特征来说, 居民低碳行为存在年龄、 职业和学历差异。年轻人趋于红色型低碳行为; 低 学历的工薪阶层日常行为趋于被迫型低碳行为。 和年轻居民相比, 老年人日常行为趋于绿色、被迫 和易感型的可能性分别为 $45.4 \% 、 78.9 \% 、 32.8 \%$, 受 生活习惯和身体情况影响, 年长者消费较为节俭, 而年轻人生活消费类型多样且以高碳化为主, 低碳 行为趋于红色型。和无业居民相比, 工薪阶层居民 低碳行为趋于被迫型。此处需说明, 为获取一般性 结论, 工薪阶层包括企业员工和自由职业者, 即使 居民的低碳认知水平较低,但由于需要承担生活压 力, 其行为也会趋向于低碳化, 因此该类型居民低 碳行为大多为被迫型。高中以下低学历居民低碳 行为趋于被迫型, 低学历居民大多为中低收人的工 薪阶层, 低碳认知水平不高, 经济条件较差, 低碳行 为趋向于被迫型。

(2) 住房面积大、收人高且机动化出行的居民 低碳行为趋于红色型。和高收人居民相比, 中等收 人居民趋于绿色型、被迫型和易感型低碳行为的可 能性分别为 $52.4 \% 、 98.8 \% 、 53.9 \%$ 。经济收人是居 民消费的前提，高收人居民的消费层次相对较高， 生活方式趋于高碳化。自行车是绿色出行的首选, 选择其为出行方式的居民低碳行为趋于绿色型, 且 选择该出行方式的居民大多需要承担生活压力, 日 常生活行为低碳化水平较高。和住房面积大的居 民相比,住房面积中等的居民趋于绿色型、被迫型 和易感型低碳行为的可能性分别 $53.1 \%$ 、 $80 \%$ 和 $39.9 \%$ 。住房面积反映居民的经济条件,经济压力 大的居民住房面积较小, 日常消费行为较为节俭, 趋于低碳化生活方式。

\section{2 建成环境}

居住区微观建成环境对居民低碳行为产生影 响。高档住宅的居民趋于易感型低碳行为; 居住在 修建于 2000-2010年住房的居民趋于被迫型低碳 行为; 高步行指数区域的居民趋于绿色型低碳行为。

(1) 住房环境影响居民低碳行为, 居住在 2000-2010年修建住房的居民为被迫型低碳行为， 高档住宅居民低碳行为大多为易感型。和 2000 年 前修建住房相比, 2000-2010年修建住房的居民趋 于被迫型低碳行为, 高档住房居民趋于易感型低碳 行为, 这是因为高档住宅居民大多经济条件较好, 生活压力相对较轻, 即便低碳认知水平高, 但日常 生活消费往往会追求舒适、便利,而趋于易感型低 
表 4 居民不同类型低碳行为的多元 Logistic 回归结果

Tab.4 Multinomial Logistic regression results of different types of low-carbon behaviors of residents

\begin{tabular}{|c|c|c|c|c|c|c|c|c|c|c|c|}
\hline 属性 & 指标 & 解释变量 & $\begin{array}{l}\text { 模型 } 1 \\
\text { 绿色型 }\end{array}$ & $\begin{array}{l}\text { 模型 } 2 \\
\text { 被迫型 }\end{array}$ & $\begin{array}{l}\text { 模型 } 3 \\
\text { 易感型 }\end{array}$ & 属性 & 指标 & 解释变量 & $\begin{array}{l}\text { 模型 } 1 \\
\text { 绿色型 }\end{array}$ & $\begin{array}{l}\text { 模型 } 2 \\
\text { 被迫型 }\end{array}$ & $\begin{array}{l}\text { 模型 } 3 \\
\text { 易感型 }\end{array}$ \\
\hline & 常量 & & $-2.332^{* *}$ & $-7.015^{* * * *}$ & $-1.583^{*}$ & & 住房 & 小 & $-0.684^{* *}$ & -0.095 & -0.213 \\
\hline 社 & 年龄 & 青年 & $-0.454^{* *}$ & $-0.789^{* * *}$ & $-0.328^{* *}$ & & 面积 & 中 & $-0.531^{* *}$ & $-0.800^{* *}$ & $-0.399^{* * *}$ \\
\hline 会 & & 老年 & $0^{\mathrm{b}}$ & $0^{\mathrm{b}}$ & $0^{\mathrm{b}}$ & & & 大 & $0^{\mathrm{b}}$ & $0^{\mathrm{b}}$ & $0^{\mathrm{b}}$ \\
\hline 经 & 职业 & 行政人员 & -0.087 & -0.035 & -0.145 & 建 & 建造 & 2010年后 & 0.132 & 0.418 & 0.033 \\
\hline 济 & & 工薪阶层 & 0.311 & $0.612^{*}$ & 0.036 & 成 & 年代 & 2000-2010年 & -0.201 & $1.073^{*}$ & -0.046 \\
\hline 属 & & 无业 & $0^{\mathrm{b}}$ & $0^{\mathrm{b}}$ & $0^{\mathrm{b}}$ & 环 & & 2000年前 & $0^{\mathrm{b}}$ & $0^{\mathrm{b}}$ & $0^{\mathrm{b}}$ \\
\hline \multirow[t]{13}{*}{ 性 } & 月收 & 低 & 0.211 & $1.030^{* *}$ & $0.788^{* * * *}$ & 境 & 住房 & 普通 & -0.164 & -0.166 & $-0.347^{*}$ \\
\hline & 人 & 中 & $0.524^{* *}$ & $0.988^{* * * *}$ & $0.539^{* * * *}$ & & 类型 & 高档 & $0^{\mathrm{b}}$ & $0^{\mathrm{b}}$ & $0^{\mathrm{b}}$ \\
\hline & & 高 & $0^{\mathrm{b}}$ & $0^{\mathrm{b}}$ & $0^{\mathrm{b}}$ & & 步行 & 高 & $0.260^{\circ}$ & 0.012 & 0.187 \\
\hline & 学历 & 高中以下 & 0.024 & $0.971^{*}$ & -0.040 & & 指数 & 低 & $0^{\mathrm{b}}$ & $0^{\mathrm{b}}$ & $0^{\mathrm{b}}$ \\
\hline & & 高中或大专 & 0.088 & 0.216 & 0.067 & 低 & 低碳 & 高 & $2.365^{* * *}$ & 0.584 & $2.107^{*+* *}$ \\
\hline & & 本科及以上 & $0^{\mathrm{b}}$ & $0^{\mathrm{b}}$ & $0^{\mathrm{b}}$ & 碳 & 行为 & 较高 & $1.522^{* *}$ & 0.540 & $1.886^{* * *}$ \\
\hline & 私家 & 无 & $1.331^{* * *}$ & $1.604^{* * * *}$ & $1.465^{* * * *}$ & 认 & 意向 & 较低 & $2.109^{* * *}$ & 0.492 & $2.110^{* * * *}$ \\
\hline & 车 & 1 辆 & $0.674^{* *}$ & 0.226 & $0.944^{* * * *}$ & 知 & & 低 & $0^{\mathrm{b}}$ & $0^{\mathrm{b}}$ & $0^{\mathrm{b}}$ \\
\hline & & 多辆 & $0^{\mathrm{b}}$ & $0^{\mathrm{b}}$ & $0^{\mathrm{b}}$ & & 认知 & 低 & -0.123 & -0.435 & -0.307 \\
\hline & 自行 & 无 & $-1.206^{* * *}$ & -0.115 & $-0.697^{* * *}$ & & 水平 & 中等 & $-0.510^{* *}$ & -0.548 & $-0.469^{* *}$ \\
\hline & 车 & 1 辆 & $-0.458^{*}$ & 0.325 & -0.281 & & & 高 & $0^{\mathrm{b}}$ & $0^{\mathrm{b}}$ & $0^{\mathrm{b}}$ \\
\hline & & 多辆 & $0^{\mathrm{b}}$ & $0^{\mathrm{b}}$ & $0^{\mathrm{b}}$ & & 认知 & 差 & 0.249 & $0.801^{* *}$ & -0.096 \\
\hline & & & & & & & 环境 & 好 & $0^{\mathrm{b}}$ & $0^{\mathrm{b}}$ & $0^{\mathrm{b}}$ \\
\hline
\end{tabular}

注: $* * * * * *$ 分别表示在 $10 \% 、 5 \%$ 和 $1 \%$ 水平上显著; $\mathrm{b}$ 为参照类。

碳行为; 而居住在普通单元式住宅的居民低碳行为 水平较高。主要原因如下: 1 住房类型侧面体现居 民的月收人、职业等相关因素, 高档住宅居民消费层 次较高, 日常生活行为趋于高碳化; (2) 普通单元式 住房大多为商品房, 受住房交易化影响, 周围各项基 础服务设施便利且混合度高, 居民趋向于低碳化 出行。

(2) 居住在高步行指数区域的居民趋于绿色型 低碳行为, 步行指数反映居民的居住环境, 高步行 指数区域周围基础设施便利、服务设施混合度较 高, 居民选择步行或自行车等出行方式就可到达目 的地。

\section{3 低碳认知}

低碳行为意向程度高和认知水平高的居民趋 于绿色型低碳行为。与行为意向程度低的居民相 比, 意向程度高的居民趋于绿色型低碳行为。低碳 行为意向指居民对低碳行为的主观判断, 反映居民 对低碳行为的认可度和接受程度, 行为意向积极的 居民低碳行为趋于绿色型。低碳认知水平中等的 居民趋于绿色型低碳行为的可能性为 $51 \%$, 低碳认 知水平作为居民对低碳行为的熟悉程度, 认知水平
高的居民低碳行为趋于绿色型。和认知环境好的 居民相比,认知环境差的居民低碳行为趋于被迫型 的可能性为 $80 \%$,认知环境代表着居住环境周围接 触低碳知识的程度和机会, 居民接触到低碳知识的 机会越大, 其低碳认知水平会提高, 无形之中会增 加低碳行为。

\section{5 结论和讨论}

\section{1 结论}

本文利用郑州市问卷调查数据, 分析各类低碳 行为的特征及影响因素, 主要结论如下:

(1) 城市化快速推进、基础设施和城市形态尚 未定型的郑州市, 会存在低碳行为一认知偏差现象, 且各类低碳行为比例差距较大。郑州市易感型居 民所占比重最大 $(46 \%)$, 被迫型居民所占比例最小 $(8 \%)$, 差距较大, 即郑州市可能有 $54 \%$ 的居民存在 低碳行为一认知偏差。

(2) 居住在土地利用混合度较高、基础设施便 利区域的居民趋于绿色型低碳行为。被迫型、易感 型和红色型低碳行为呈现随机分布, 而绿色型低碳 
行为居民主要聚集在二七广场、郑东新区、郑州东 站等土地利用混合度较高、基础设施便利区域。

(3) 4 种低碳行为影响因素差异较大。居住区 步行指数较高、低碳认知水平高的居民趋于绿色型 低碳行为, 居民低碳行为与低碳认知水平均较高。 居住区住房条件相对较差、居民学历水平低, 居民 趋于被迫型低碳行为, 低碳认知水平低但低碳行为 水平较高。高档住宅居民趋于易感型低碳行为, 居 民的低碳认知水平高但低碳行为水平较低。经济 水平高的年轻居民趋于红色型低碳行为, 低碳行为 和低碳认知水平均较低。

\section{2 讨论}

相较以往仅涉及单个研究对象, 本文在彩色编 码基础下从低碳行为-认知 2 个维度出发, 将居民低 碳行为分为 3 种颜色 4 种类型, 为低碳城市建设提 供新的切人点, 更有利于低碳城市建设; 此外构建 “空间-行为/认知” 分析框架, 使用多元 Logistic 回 归从社会经济属性、低碳认知和建成环境方面分析 各类低碳行为的影响因素。基于此,本文提出以下 几点建议:

(1) 因人而异改善居民生活方式。(1) 针对经济 条件好、消费层次高的红色型居民, 不仅需要政府 主导改善居住区的建成环境和基础设施条件,社区 还要辅助管理宣传来提高居民低碳认知水平。(2) 对于日常生活行为易考虑舒适、便利, 偶尔实施绿 色行为的高档住宅居民, 需要凸显出绿色型低碳行 为的优势, 来减少低碳行为一认知差距。(3) 居住在 2000-2010年修建住房的低学历工薪阶层居民, 大 多为被迫型居民(即低碳认知水平较低, 但由于经 济压力大而低碳行为水平较高), 不能仅局限于低 碳知识宣传,还要给予相应的补贴和奖励政策。

(2) 因地制宜进行老城区改造。一方面, 结合 老城区所处位置, 对老城区重新规划定位, 加强居 住区周围有购物、医疗、娱乐、教育等设施, 保证微 观居住环境对居民低碳生活行为的引导, 减少该区 域居民的低碳行为-认知差距; 另一方面, 向该区域 居民提供低碳宣讲和教育, 提高低碳城市建设的公 众参与度。

(3) 调整城市设施规划布局。优化城市各项服 务设施的分区规划, 解决规划中存在的居民职住分 离、供需失衡等问题; 并提高城市土地利用混合度、 道路便利度及可达性, 增加居民的通行效率。

本文仍存在一些不足之处: (1) 数据获取方面,
本文主要数据来自于问卷调查数据, 不可避免会受 到一些主观不确定性因素影响, 在后续研究中可与 居民的 GPS 行为轨迹数据相结合进行数据篮选; (2) 理论分析方面:郑州市居民可能会根据自己的社 会经济特征、工作属性和偏好来选择日常生活行 为, 存在一定自选择效应, 因此居民的居住、通勤、 休闲娱乐、个人事务等各类活动的属性特征均会影 响居民低碳行为, 本文仅侧重于从微观居住环境分 析, 尚未从自选择视角综合各类属性来分析; (3) 职 业分类方面,为获得一般性的结论而将企业员工和 自由职业者笼统地划分为工薪阶层, 而企业员工和 自由职业者的影响程度有可能会存在不同,这些都 需要进行后续细化研究。

\section{参考文献(References)}

[1] Edenhofer O, Pichs-Madruga R, Sokona Y, et al. IPCC. Climate change 2014: Mitigation of Climate Change: Working Group III Contribution to the Fifth Assessment Report of the Intergovernmental Panel on Climate Change [M]. Cambridge, UK: Cambridge University Press, 2014.

[2] 王少剑, 苏泳涃, 赵亚博. 中国城市能源消费碳排放的区 域差异、空间溢出效应及影响因素 [J]. 地理学报, 2018, 73(3): 414-428. [Wang Shaojian, Su Yongxian, Zhao Yabo. Regional inequality, spatial spillover effects and influencing factors of China's city-level energy-related carbon emissions. Acta Geographica Sinica, 2018, 73(3): 414428. ]

[3] Gadenne D, Sharma B, Kerr D, et al. The influence of consumers' environmental beliefs and attitudes on energy saving behaviours [J]. Energy Policy, 2011, 39(12): 76847694.

[4] 王晓磊. 内蒙古自治区碳排放峰值预测及综合控制策略 研究 [D]. 北京: 中国地质大学, 2017. [Wang Xiaolei. The study on carbon emission peck forecast and its comprehensive control strategies of Inner Mongolia. Beijing, China: China University of Geosciences, 2017. ]

[5] Li J, Zhang D Y, Su B. The impact of social awareness and lifestyles on household carbon emissions in China [J]. Ecological Economics, 2019, 160: 145-155.

[6] Geng J C, Long R Y, Chen H, et al. Exploring the motivation-behavior gap in urban residents' green travel behavior: A theoretical and empirical study [J]. Resources, Conservation and Recycling, 2017, 125: 282-292.

[7] Ding Z H, Jiang X, Liu Z H, et al. Factors affecting lowcarbon consumption behavior of urban residents: A com- 
prehensive review $[\mathrm{J}]$. Resources, Conservation and Recycling, 2018, 132: 3-15.

[8] 潘丽丽, 王晓宇. 基于主观心理视角的游客环境行为意 愿影响因素研究: 以西溪国家湿地公园为例 [J]. 地理科 学, 2018, 38(8): 1337-1345. [Pan Lili, Wang Xiaoyu. The factors affecting the intention to exhibit environmental behavior by tourists: A case study of Xixi National Wetland Park in Hangzhou. Scientia Geographica Sinica, 2018, 38 (8): 1337-1345. ]

[9] Yang S, Zhang Y B, Zhao D T. Who exhibits more energysaving behavior in direct and indirect ways in china? The role of psychological factors and socio-demographics [J]. Energy Policy, 2016, 93: 196-205.

[10] Ding Z H, Wang G Q, Liu Z H, et al. Research on differences in the factors influencing the energy-saving behavior of urban and rural residents in China: A case study of Jiangsu Province [J]. Energy Policy, 2017, 100: 252259.

[11] Ye H, Ren Q, Hu X Y, et al. Low-carbon behavior approaches for reducing direct carbon emissions: Household energy use in a coastal city [J]. Journal of Cleaner Production, 2017, 141: 128-136.

[12] 高志刚, 李金倳, 艳. 乌鲁木齐市社区居民低碳意识与 行为影响因素研究: 基于结构方程的分析 [J]. 新疆大 学学报(哲学・人文社会科学版), 2017, 45(4): 1-9. [Gao Zhigang, Li Xin, Zhang Yan. On the influential factors of low-carbon consciousness and behavior of Urumqi residents: An analysis of structural equation. Journal of Xinjiang University (Philosophy, Humanities \& Social Sciences), 2017, 45(4): 1-9. ]

[13] Poruschi L, Ambrey C L. On the confluence of city living, energy saving behaviours and direct residential energy consumption $[\mathrm{J}]$. Environmental Science and Policy, 2016, 66: 334-343.

[14] Ramos A, Labandeira X, Löschel A. Pro-environmental households and energy efficiency in Spain $[\mathrm{J}]$. Environmental and Resource Economics, 2016, 63(2): 367-393.

[15] 曲英. 城市居民生活垃圾源头分类行为研究 [D]. 大连: 大连理工大学, 2007. [Qu Ying. The study on residential behavior for source separation of household waste. Dalian, China: Dalian University of Technology, 2007. ]

[16] 王国猛, 黎建新, 廖水香, 等. 环境价值观与消费者绿色 购买行为: 环境态度的中介作用研究 [J]. 大连理工大 学学报 (社会科学版), 2010, 31(4): 37- 42. [Wang Guomeng, Li Jianxin, Liao Shuixiang, et al. Study of the relationship between environmental values and green purchasing behavior: The mediating effect of environmental attitude. Journal of Dalian University of Technology (So- cial Sciences), 2010, 31(4): 37-42. ]

[17] Pothitou M, Hanna R F, Chalvatzis K J. Environmental knowledge, pro-environmental behaviour and energy savings in households: An empirical study [J]. Applied Energy, 2016, 184: 1217-1229.

[18] Mills B, Schleich J. What's driving energy efficient appliance label awareness and purchase propensity [J]. Energy Policy, 2010, 38(2): 814-825.

[19] Allcott H. Social norms and energy conservation [J]. Journal of Public Economics, 2011, 95(9-10): 1082-1095.

[20] Shi H X, Fan J, Zhao D T. Predicting household PM PM.seduction behavior in Chinese urban areas: An integrative model of theory of planned behavior and norm activation theory [J]. Journal of Cleaner Production, 2017, 145: 6473.

[21] Bai Y, Liu Y. An exploration of residents' low-carbon awareness and behavior in Tianjin, China [J]. Energy Policy, 2013, 61: 1261-1270.

[22] 洪学婷, 张宏梅. 国外环境责任行为研究进展及对中国 的启示 [J]. 地理科学进展, 2016, 35(12): 1459-1471. [Hong Xueting, Zhang Hongmei. Progress of environmentally responsible behavior research and its enlightenment to China. Progress in Geography, 2016, 35(12): 1459-1471. ]

[23] 柴彦威, 谭一洺, 申悦. 空间-行为互动理论构建的基本 思路 [J]. 地理研究, 2017, 36(10): 1959-1970. [Chai Yanwei, Tan Yiming, Shen Yue. Space-behavior interaction theory: Basic thinking of general construction. Geographical Research, 2017, 36(10): 1959-1970. ]

[24] 李琬, 但波, 孙斌栋, 等. 轨道交通对出行方式选择的影 响研究: 基于上海市 80 后微观调查样本的实证分析 [J]. 地理研究, 2017, 36(5): 945-956. [Li Wan, Dan Bo, Sun Bindong, et al. The influence of rail transit accessibility on the shift of travel modal choice: Empirical analysis based on the micro survey of the 1980s generation in Shanghai. Geographical Research, 2017, 36(5): 945956. ]

[25] 周素红, 宋江宇, 宋广文. 广州市居民工作日小汽车出 行个体与社区双层影响机制 [J]. 地理学报, 2017, 72 (8): 1444- 1457. [Zhou Suhong, Song Jiangyu, Song Guangwen, et al. Examining the dual- levels impact of neighbourhood and individual variables on car use on weekdays in Guangzhou. Acta Geographica Sinica, 2017, 72(8): 1444-1457. ]

[26] Rokeach M. The nature of human values [M]. New York, USA: The Free Press, 1973.

[27] 吴春梅, 张伟. 居民低碳认知态度与行为的实证研究 [J]. 技术经济与管理研究, 2013(7): 123-128. [Wu Chun- 
mei, Zhang Wei. An empirical study of low-carbon cognitive, attitudes and behaviors. Technoeconomics and Management Research, 2013(7): 123-128. ]

[28] 党宁, 吴必虎, 张雯霞. 计划行为还是理性行为? 上海居 民近城游憩行为研究 $[\mathrm{J}]$. 人文地理, 2017, 32(6): 137145. [Dang Ning, Wu Bihu, Zhang Wenxia. Planned behavior or reasoned action? The study on Shanghai residents' suburban recreation behaviors. Human Geography, 2017, 32(6): 137-145. ]

[29] 白凯, 李创新, 张翠娟. 西安城市居民绿色出行的群体 参照影响与自我价值判断 [J]. 人文地理, 2017, 32(1): 37-46. [Bai Kai, Li Chuangxin, Zhang Cuijuan. Reference group influence and self-perceived value judgment of Xi'an urban residents' green travel behavior. Human Geography, 2017, 32(1): 37-46. ]

[30] 张红霞, 苏勤, 陶玉国. 住宿业节能减碳研究进展及启 示 [J]. 地理科学进展, 2017, 36(6): 774- 783. [Zhang Hongxia, Su Qin, Tao Yuguo. Research progress in energy saving and carbon emission reduction research of the tourist accommodation industry. Progress in Geography, 2017, 36(6): 774-783. ]

[31] 赵燕慧, 路紫, 白龙. 心理地理学视角下空难暴露人群 心理应激机制分析及其理论延伸 [J]. 人文地理, 2018, 33(3): 20-26. [Zhao Yanhui, Lu Zi, Bai Long. Phychological stress mechanism of air crash exposed population from the perspective of psychogeography and its theoretical extension. Human Geography, 2018, 33(3): 20-26. ]

[32] Guagnano G A, Stern P C, Dietz T. Influences on attitudebehavior relationships: A natural experiment with curbside recycling $[\mathrm{J}]$. Environment and Behavior, 2000, 56 (3): 407-424.

[33] 曹小曙, 梁斐雯, 陈慧灵. 特大城市空间形态差异对交 通网络效率的影响 [J]. 地理科学, 2019, 39(1): 41-51. [Cao Xiaoshu, Liang Feiwen, Chen Huiling. Influence of different spatial forms for metropolitans on transportation network efficiency. Scientia Geographica Sinica, 2019, 39(1): 41-51. ]

[34] Bereitschaft B, Debbage K. Urban form, air pollution, and $\mathrm{CO}_{2}$ emissions in large US metropolitan areas $[\mathrm{J}]$. The Professional Geographer, 2013, 65(4): 612-635.

[35] Ou J P, Liu X P, Li X, et al. Quantifying the relationship between urban forms and carban emissions using panel data analysis [J]. Landscape Ecology, 2013, 28(10): 1889-1907.

[36] 张纯. 城市社区形态与再生 [M]. 南京: 东南大学出版 社, 2013. [Zhang Chun. Community urban form and regeneration. Nanjing, China: Southeast University Press, 2013. ]
[37] 吴巍, 宋彦, 洪再生, 等. 居住社区形态对住宅能耗影响 研究: 以宁波市为例 [J]. 城市发展研究, 2018, 25(1): 15-20, 28. [Wu Wei, Song Yan, Hong Zaisheng, et al. Research of the influence of community form on residential energy consumption: A case study on Ningbo City. Urban Development Studies, 2018, 25(1): 15-20, 28. ]

[38] 刘清春, 张芗芗, 肖燕, 等. 济南市主城区私家车日常出 行碳排放特征及影响因素 [J]. 资源科学, 2018, 40(2): 262-272. [Liu Qingchun, Zhang Yingying, Xiao Yan, et al. Characteristics and determinants of carbon emissions from daily private cars travel in central area of Jinan. Resources Science, 2018, 40(2): 262-272. ]

[39] 赵宏波, 冯渊博, 董冠鹏, 等. 大城市居民自评健康与环 境危害感知的空间差异及影响因素: 基于郑州市区的 实证研究 [J]. 地理科学进展, 2018, 37(12): 1713-1726. [Zhao Hongbo, Feng Yuanbo, Dong Guanpeng, et al. Spatial differentiation and influencing factors of residents' self-rated health and environmental hazard perception: A case study of Zhengzhou City. Progress in Geography, 2018, 37(12): 1713-1726. ]

[40] 杨波. 郑州市居民对低碳商品的认知状况和消费意愿 影响因素分析: 基于居民调查数据的实证研究 [J]. 经 济经纬, 2012(1): 122-126. [Yang Bo. An analysis of Zhengzhou urban residents' cognition of low carbon commodities and factors affecting consumption desires: An empirical study based on resident survey data. Economic Survey, 2012(1): 122-126. ]

[41] 荣培君, 张丽君, 秦耀辰, 等. 城市居民嵌人式碳排放空 间差异及影响因素 [J]. 经济地理, 2018, 38(6): 178186. [Rong Peijun, Zhang Lijun, Qin Yaochen, et al. The spatial differences and influencing factors of urban residential embedded carbon emissions. Economic Geography, 2018, 38(6): 178-186. ]

[42] 周垠, 龙瀛. 街道步行指数的大规模评价: 方法改进及 其成都应用 [J]. 上海城市规划, 2017(1): 88-93. [Zhou Yin, Long Ying. Large-scale evaluation for street walkability: Methodological improvements and the empirical application in Chengdu. Shanghai Urban Planning Review, 2017(1): 88-93. ]

[43] 张丽君, 孙芗芗, 郑智成, 等. 城市社区日常服务设施可 步行性评价: 以开封市为例 [J]. 城市观察, 2018(1): 133-144. [Zhang Lijun, Sun Yingying, Zheng Zhicheng, et al. Urban neighborhood walkability assessment of daily service facilities: A case study of Kaifeng. Urban Insight, 2018(1): 133-144. ]

[44] 袁媛, 林静, 谢磊. 近 15 年来国外居民健康的邻里影响 研究进展: 基于 CiteSpace 软件的可视化分析 [J]. 热带 地理, 2018, 38(3): 440-450. [Yuan Yuan, Lin Jing, Xie 
Lei. Review of the research in foreign countries in the past 15 years about neighborhood effects on residents' health: A visualized analysis based on CiteSpace software. Tropical Geography, 2018, 38(3): 440-450. ]

[45] 塔娜, 柴彦威, 关美宝. 北京郊区居民日常生活方式的
行为测度与空间一行为互动 $[\mathrm{J}]$. 地理学报, 2015, 70 (8): 1271-1280. [Ta Na, Chai Yanwei, Guan Meipo. Suburbanization, daily lifestyle and space-behavior interaction in Beijing. Acta Geographica Sinica, 2015, 70(8): 1271-1280. ]

\title{
Influencing factors of low-carbon behaviors of residents in Zhengzhou City from the perspective of cognition-behavior gaps
}

\author{
ZHANG Jingfei ${ }^{1,2}$, ZHANG Lijun $^{1,2^{*}}$, QIN Yaochen ${ }^{1,2}$, WANG Xia ${ }^{1,2}$, \\ SUN Yingying ${ }^{1,2}$, RONG Peijun ${ }^{3}$ \\ (1. Key Laboratory of Geospatial Technology for Middle and Lower Yellow River Regions, Kaifeng 475004, Henan, China; \\ 2. College of Environment and Planning, Hennan University, Kaifeng 475004, Hennan, China; \\ 3. College of Tourism and Exhibition, Henan University of Economics and Law, Zhengzhou 450052, China)
}

\begin{abstract}
Residents' low-carbon behavior-cognition relationship has been widely concerned by scholars since cognition- behavior separation is a key factor that hinders the construction of low-carbon cities and the transformation of residents' lifestyle. This study constructed a spatial-behavioral/cognitive analysis framework and used large-scale field survey and remote sensing data of Zhengzhou City in 2018, as well as expert scoring method to estimate the low-carbon behavior level and low-carbon cognition level of 1485 families based on their daily travel and consumption of household energy. The color coding method was used to classify their behaviors into the green type, the forced type, the susceptible type, and the red type based on cognition-behavior gaps, and the multiple Logistic regression model was used to analyze the influencing factors of different low-carbon behaviors. The research findings show that: 1) There are large cognition-behavior gaps in low-carbon behaviors of residents in Zhengzhou City, and the proportions of the four low-carbon behaviors vary greatly. 2) Green lowcarbon behaviors are mainly concentrated in areas with highly mixed land use and convenient infrastructure, and forced low-carbon behaviors, susceptible low-carbon behaviors, and red low-carbon behaviors are randomly distributed in space. 3) The influencing factors of the four types of low-carbon behaviors are very different. Residents living in areas with a high walking index score and a high level of low-carbon cognition tend to exhibit green low-carbon behaviors, and have a high level of low-carbon behavior and low-carbon cognition. In areas where housing conditions are relatively poor and residents have low levels of education, the residents tend to exhibit forced low-carbon behaviors, their low-carbon cognition level is low but low-carbon behavior level is high. Residents of high-end residential buildings tend to show susceptible low-carbon behaviors, they have a high level of low-carbon cognition but a low level of low-carbon behaviors. Young residents with high economic status tend to exhibit red low-carbon behaviors and have a low level of low-carbon behavior and low-carbon cognition.
\end{abstract}

Keywords: low-carbon behavior; built environment; behavior-cognition gap; multinomial Logistic regression; Zhengzhou City 